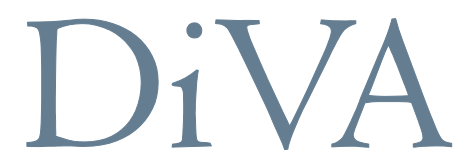

http://uu.diva-portal.org

This is an author-produced version. It does not include the final publisher proof-corrections or pagination.

Citation for the published Book Chapter:

Koponen, $\mathrm{V}$.

"Some Connections Between Finite and Infinite Model Theory" In:

Esparza, J; Michaux, C; Steinhorn, C. (eds.)

"Finite and Algorithmic Model Theory"

London Mathematical Society Lecture Note Series, Number 379. ISSN: 0076-0552

Cambridge University Press, 2011, pp. 109-139

ISBN: 9780521718202

URL: http://www.cambridge.org/se/knowledge/isbn/item5634823/?site locale=sv SE

Access to the published version may require subscription. 


\title{
SOME CONNECTIONS BETWEEN FINITE AND INFINITE MODEL THEORY
}

\author{
VERA KOPONEN
}

\section{INTRODUCTION}

Most of the work in model theory has, so far, considered infinite structures and the methods and results that have been worked out in this context can usually not be transferred to the study of finite structures in an obvious way. In addition, some basic results from infinite model theory fail within the context of finite models. The theory about finite structures has largely developed in connection with theoretical computer science, in particular complexity theory [12]. The question arises whether these two "worlds", the study of infinite structures and the study of finite structures, can be weaved together in some way and enrich each other. In particular, one may ask if it is possible to adapt notions and methods which have played an important role in infinite model theory to the context of finite structures, and in this way get a better understanding of fairly large and sufficiently well-behaved classes of finite structures.

If we are to study structures in relation to some formal language, then the question arises which one to choose. Most of infinite model theory considers first-order logic. Within finite model theory various restrictions and extensions of first-order logic have been considered, since first-order logic may be considered as being both too strong and too weak (in different senses) for the study of finite structures. A reasonable candidate for studying finite structures, with a viewpoint from infinite model theory, is the language $L^{n}$, first order logic $L$ restricted to formulas in which at most $n$ variables occur, whether free or bound. Theories consisting of only $L^{n}$-formulas, even those which are "complete" within $L^{n}$, may have both finite and infinite models, or only finite models, or only infinite models. The language $L^{n}$ has the nice properties of being closed under subformulas, quantification and negation. Also, there is a pebble game which distinguishes whether two structures satisfy exactly the same $L^{n}$-sentences or not ([23] and implicitly in [29]).

The notion of a type plays an important role in infinite model theory. In finite model theory the notion of an $L^{n}$-type, i.e. a type restricted to $L^{n}$-formulas, has been used; the number of different $L^{n}$-types of an $L^{n}$-theory can be seen as a measure of the complexity of the theory. Dawar observed [5] that for every $L^{n}$-theory $T$ with finite models there is an upper bound, depending only on the number of $L^{n}$-types (in $n$ free variables) of $T$, of the size of the smallest model of $T$. Later Grohe proved that this upper bound is not recursive [17]. The language $L^{n}$ has also been considered in the context of (only) infinite models in the work of Hedman [19] where complete theories (within full first-order logic) which are axiomatizable by $L^{n}$-sentences are studied. For a general overview about interactions (and differences) between finite and infinite model theory, see [30]. For a survey about the use of finite variable logics in finite model theory, see [16].

Within infinite model theory the area of stability theory has had great influence. It studies a quite large class of "managable" (infinite) structures and their complete firstorder theories. Work in the direction of developing the basics of a similar theory for finite structures was first carried out by Hyttinen [21]. Then, from a different viewpoint, the author developed some results, inspired by stability theory, aiming at understanding when an $L^{n}$-theory with infinite models also must have arbitrarily large finite models $[9,8]$. 
Further developments in this direction where made by Baldwin and Lessmann [2] and by Hyttinen [22]. For an overview, with a historical perspective, of finite and infinite model theory and recent interactions between them, see [1].

Another approach to undestanding certain finite and countably infinite structures culminates with the work about smoothly approximable structures in [3]. This line of research started with Lachlan's work on stable finitely homogeneous structures (surveyed in [27]) and Zilber's work on totaly categorical structures [33]. It continued with joint work by Cherlin, Harrington and Lachlan on $\omega$-categorical $\omega$-stable structures [4] and then with the work of Kantor, Liebeck and Macpherson [24], to reach its current state in [3]. Smoothly approximable structures are infinite but can be approximated by "nicely embedded" finite structures which, intuitively speaking, are quite "homogeneous" or "regular". The theory of smoothly approximable structures can also be seen as a study of finite structures with few types.

More recently, a direction of research initiated by Macpherson and Steinhorn [28] and continued by Elwes $[13,14]$ studies classes of finite structures in which definable sets have a uniform asymptotic behaviour, as the cardinalities of the universes increase. The complete theory $T$ of a non-principal ultraproduct of such a class of finite structures (called an 'asymptotic class') is simple with finite SU-rank and there is a notion of measure on the definable subsets of models of $T$, but $T$ is not necessarily smoothly approximable. See [15] for a survey of the topic.

In this article an overview is given of a line of research which considers $L^{n}$-theories with infinite models and tries to isolate conditions for when these have arbitrarily large finite models and when least upper bounds for the smallest model is recursive in terms of the number of $L^{n}$-types in $n$ free variables. Although some results are stated within a more general context, considering some arbitrary fraction of first-order logic which is closed under subformulas, and some results could be stated in a somewhat more general way, we mostly stick to the language $L^{n}$ for the sake of simplicity. Moreover, when working with $L^{n}$ we usually consider an $L^{n}$-theory $T$ such that $T$ is finitely axiomatizable in $L^{n}$ and complete (within $L^{n}$ ) in the sense that for every $\varphi \in L^{n}, T \models \varphi$ or $T \models \neg \varphi$. The motivation is that we like to find conditions for $T$ which imply that $T$ has a finite model, and facts 1.6 and 1.7 below imply the following: if $T$ is an $L^{n}$-theory and no complete $L^{n}$-theory $T^{\prime} \supseteq T$ exists such that $T^{\prime}$ is finitely axiomatizable, then $T$ has no finite model.

The basic idea is to isolate conditions for a finitely axiomatizable complete $L^{n}$-theory $T$ which guarantee the existence of a model $M$ of $T$ which is smoothly approximable, since such an $M$ has the property that every sentence which is true in $M$ is true in arbitrarily large finite substructures of $M$. Moreover, in this situation the theory of smoothly approximable structures implies that a recursive upper bound, in terms of the number of $L^{n}$-types in $n$ free variables, of the smallest model exists (in contrary to the general situation, as proved in [17]).

Sections $1-7$ of this article try to unify, as much as possible, the approaches of [9], [8] and [2]. Hyttinen's paper [22] on canonical finite diagrams and quantifier elimination is highly recommended since it develops, in a more general context, part of the theory and several of the results. Here I have chosen to expose the subject via a more "down-to-earth"-approach focused on $L^{n}$-theories, although some generality is lost.

Sections 5 and 6 discusses infinite structures which have the finite model property but which are not necessarily smoothly approximable (the random bipartite graph is an example [24]). This may be useful for understanding other classes of $L^{n}$-theories than those treated in earlier sections. The last section contains a list of questions and problems. 


\section{Preliminaries}

1.1. Finite variable logic. In this section we introduce the language $L^{n}$, the subset of $L$ containing all formulas in which at most $n$ distinct variables occur.

Definition 1.1. (i) Let $V=\left\{v_{1}, v_{2}, v_{3}, \ldots\right\}$ be the set of variables which are used in formulas of $L$.

(ii) By $x, y, z, \bar{x}, \bar{y}, \bar{z}$, sometimes with indices, we denote variables and finite sequences of variables. Similarly, $a, b, c, \bar{a}, \bar{b}, \bar{c}$ denote elements and finite sequences of elements from structures. When writing $\bar{a} \in A$ we mean that every element of the sequence $\bar{a}$ belongs to $A$. If, in addition, we like to stress that $\bar{a}$ has length $n$, then we may write $\bar{a} \in A^{n}$.

(iii) $L$ always denotes the set of all first-order formulas over some vocabulary (or signature). We always assume that $L$ is countable.

(iv) If a formula in $L$ is denoted by $\varphi(\bar{x})$ then we mean that every free variable in that formula belongs to the sequence $\bar{x}$.

(v) For any $n<\omega, L^{n}$ denotes the set of all formulas $\varphi(\bar{x}) \in L$ such that at most $n$ distinct variables occur in $\varphi(\bar{x})$ (whether bound or free). We allow $\bar{x}$ to contain "dummy variables" (not occuring in the formula denoted by $\varphi(\bar{x})$ ). For example, the formula $v_{1}=v_{2} \vee v_{2}=v_{3}$ may be denoted by $\varphi\left(v_{1}, v_{2}, v_{3}, v_{4}\right)$ and consequently $\varphi\left(v_{1}, v_{2}, v_{3}, v_{4}\right) \in L^{3}$, because only three variables actually occur in the formula $\varphi\left(v_{1}, v_{2}, v_{3}, v_{4}\right)$.

(vi) An $L^{n}$-theory is a set of sentences from $L^{n}$.

(vii) An $L^{n}$-theory $T$ is called a complete $L^{n}$-theory if for every sentence $\varphi \in L^{n}, T \models \varphi$ or $T \models \neg \varphi$. Of course, a 'complete $L^{n}$-theory' need not be complete with respect to $L$.

(viii) If $M$ is an $L$-structure let

$$
T h_{L^{n}}(M)=\left\{\varphi \in L^{n}: \varphi \text { is a sentence and } M \models \varphi\right\} .
$$

So $T h_{L^{n}}(M)$ is always a complete $L^{n}$-theory.

Remark 1.2. We have not fixed $n$ special variables to be used in formulas of $L^{n}$, but we only say that at most $n$ distinct variables may occur in a formula of $L^{n}$. For instance, a formula of $L^{n}$ may contain variables among $v_{1}, \ldots, v_{n}$ or variables among $v_{n+1}, \ldots, v_{2 n}$. For example, both $v_{1}=v_{2} \vee v_{2}=v_{3}$ and $v_{2}=v_{3} \vee v_{3}=v_{4}$ belong to $L^{3}$.

We are interested in finding conditions under which an $L^{n}$-theory with infinite models also has (arbitrarily large) finite models. So we first give some easy examples showing that $L^{n}$-theories may have only infinite models, only finite models or both infinite and finite models

Examples 1.3. (a) Let $M=(\mathbb{N}, S)$, where $S$ is the successor function (or relation). Then $T h_{L^{3}}(M)$ has no finite model.

(b) If $M=(\mathbb{Q},<)$ then $T h_{L^{3}}(M)$ has no finite model.

(c) Let $M$ be a finite graph such that $v_{1}, \ldots, v_{m}$ lists all vertices of $M$ and there is an edge between $v_{i}$ and $v_{j}$ if and only if $j=i+1$ or $i=j+1$. Then every model of $T h_{L^{3}}(M)$ is isomorphic to $M$.

(d) Let $M$ be an infinite tree such that for some $m<\omega$, no path in $M$ has length $m$. Then $T h_{L^{n}}(M)$ has arbitrarily large finite models, for any $n$.

(e) Let $K$ be a finite field. Let $T$ be a set of sentences which expresses the axioms of a $K$-vector space. With scalar multiplication and vector addition represented by function symbols we may assume that $T$ is an $L^{3}$-theory. With scalar multiplication and vector addition represented by relation symbols we may assume that $T$ is an $L^{7}$-theory. $T$ has arbitrarily large finite models and hence infinite models.

1.2. Types. The notion of a 'type' plays an important role in model theory. Here we will in particular be interested in certain types which are restricted to formulas of some 
sublanguage of the first-order language $L$. We first give some definitions with associated notation and then state a few well-known results concerning types.

Definition 1.4. (i) Let $\Phi \subseteq L$, let $M$ be an $L$-structure and let $A \subseteq M$.

(ii) Define

$$
T h_{\Phi}(M, A)=\{\varphi(\bar{a}): \varphi(\bar{x}) \in \Phi, \bar{a} \in A, M \models \varphi(\bar{a})\},
$$

and let $T h_{\Phi}(M)=T h_{\Phi}(M, \emptyset)$. So $T h_{\Phi}(M)$ is the set of sentences in $\Phi$ that are true in $M$.

(iii) For a sequence of variables $\bar{x}$ we define

$$
\Phi_{\bar{x}}(A)=\{\varphi(\bar{x}, \bar{a}): \varphi(\bar{x}, \bar{y}) \in \Phi, \bar{a} \in A\},
$$

and $\Phi_{\bar{x}}=\Phi_{\bar{x}}(\emptyset)$.

(iv) A $\Phi$-type over $A$ (with respect to $T h_{\Phi}(M, A)$ ) in the free variables $\bar{x}$ is a set $p(\bar{x}) \subseteq$ $\Phi_{\bar{x}}(A)$ such that $p(\bar{x}) \cup T h_{\Phi}(M, A)$ is consistent.

(v) A $\Phi$-type $p(\bar{x})$ over $A$ is called a complete $\Phi$-type over $A$ if whenever $\varphi(\bar{x}, \bar{a}) \in \Phi_{\bar{x}}(A)$ then $\varphi(\bar{x}, \bar{a}) \in p(\bar{x})$ or $\neg \varphi(\bar{x}, \bar{a}) \in p(\bar{x})$.

(vi) $S_{m}^{\Phi}(A, M)$ is the set of all complete $\Phi$-types over $A$ (with respect to $T h_{\Phi}(M, A)$ ) in the free variables $v_{1}, \ldots, v_{m}$. If $\Phi=L$ then we may omit it.

(vii) We write $S_{m}^{n}(A, M)$ instead of $S_{m}^{L^{n}}(A, M)$.

(viii) For a complete $L^{n}$-theory $T$ we define $S_{m}^{n}(T)=S_{m}^{n}(\emptyset, M)$, where $M$ is any model of $T$ (so $T h_{L^{n}}(M, \emptyset)=T$ ). By Lemma 1.2 in [9] this definition does not depend on the choice of the model $M$ of $T$.

Below are a few facts about $L^{n}$-types.

Fact 1.5. For any complete $L^{n}$-theory $T, S_{n}^{n}(T)$ is finite if and only if there are only finitely many $L^{n}$-formulas in the free variables $v_{1}, \ldots, v_{n}$ up to equivalence modulo $T$.

The previous fact is a consequence of the "Stone duality theorem for boolean algebras" [20], but it can also be derived in a straightforward way from the definitions.

The next fact can be extracted from the proof of a similar result in [6], and it is also mentioned in [29] (in Exercise 4).

Fact 1.6. (Dawar, Lindell, Weinstein; Poizat) Suppose that the vocabulary of L is finite and contains no function symbols. If $T$ is a complete $L^{n}$-theory and $S_{n}^{n}(T)$ is finite then there is $\varphi \in L^{n}$ that axiomatizes $T$ (i.e. $\varphi \models T$ and $T \models \varphi$ ). Moreover, we can choose $\varphi$ so that its quantifier rank is at most $\left|S_{n}^{n}(T)\right|+n$.

The next fact is easy to prove, but a proof can also be found in [9].

Fact 1.7. (i) If a complete $L^{n}$-theory $T$ has a finite model $M$ then $\left|S_{n}^{n}(T)\right| \leq|M|^{n}$. (ii) If $T$ is a complete $L^{n}$-theory and $S_{n}^{n}(T)$ is infinite then $T$ has no finite models.

Using Fact 1.7, when looking for finite models of a complete $L^{n}$-theory $T$ we can rule out the case when $S_{n}^{n}(T)$ is infinite.

\subsection{Closure maps.}

Definition 1.8. Let $M$ be an $L$-structure.

(i) We call a function $\mathrm{cl}: \mathcal{P}(M) \rightarrow \mathcal{P}(M)$ a closure map (or closure function) if whenever $A \subseteq B \subseteq M$ then $A \subseteq \operatorname{cl}(A), \operatorname{cl}(\operatorname{cl}(A))=\operatorname{cl}(A)$ and $\operatorname{cl}(A) \subseteq \operatorname{cl}(B)$.

(ii) If $\operatorname{cl}(A)=A$ then we say that $A$ is closed. A sequence is closed if the set of elements occuring in the sequence is closed.

A few examples of closure maps are given below:

Examples 1.9. (a) If $\operatorname{cl}(A)=A$ for every $A \subseteq M$ then $\mathrm{cl}$ is a closure map; we say that such a closure map is trivial.

(b) If, for every $A \subseteq M, \operatorname{cl}(A)$ is the substructure of $M$ which is generated by $A$, then $\mathrm{cl}$ is 
a closure map.

(c) If, for every $A \subseteq M, \operatorname{cl}(A)$ is the algebraic closure of $A$, in the model theoretic sense, then $\mathrm{cl}$ is a closure map. See [20] for a definition of 'algebraic closure' in the model theoretic sense.

(d) If $M$ is an algebraically closed field and $\operatorname{cl}(A)$ is the algebraic closure of $A$, in the sense of field theory, then $\mathrm{cl}$ is a closure map.

(e) If $M$ is a vector space and $\operatorname{cl}(A)$ is the linear span of $A$, then $\mathrm{cl}$ is a closure map.

\section{Amalgamation Classes}

In order to prove that arbitrarily large finite models of a finitely axiomatizable theory $T$ exist we prove that a particularly nice model $M$ of $T$ exists. This $M$ will have the property that every sentence which is true in $M$ is true in a finite substructure of it. Such an $M$ exists if there is an "amalgamation class" of models of $T$ and all models in the amalgamation class are "stable". The definition of an amalgamation class is given in this section (Definition 2.5) and the notion of 'stability' is treated in Section 3.

Definition 2.1. Let $\Phi \subseteq L$ and let $M$ and $N$ be $L$-structures.

(i) If $a_{i} \in M, b_{i} \in N$, for $i<\lambda$, then we write

$$
\left(M,\left(a_{i}: i<\lambda\right)\right) \equiv_{\Phi}\left(N,\left(b_{i}: i<\lambda\right)\right)
$$

if for every $m<\omega$, every $\varphi\left(x_{1}, \ldots x_{m}\right) \in \Phi$ and every $\left\{i_{1}, \ldots, i_{m}\right\} \subseteq \lambda$,

$$
M \models \varphi\left(a_{i_{1}}, \ldots, a_{i_{m}}\right) \text { if and only if } N \models \varphi\left(b_{i_{1}}, \ldots, b_{i_{m}}\right) .
$$

(ii) A function $f: A \rightarrow N$, where $A \subseteq M$, is called a $\Phi$-elementary embedding if for every $\varphi(\bar{x}) \in \Phi$ and $\bar{a} \in A$ with $|\bar{a}|=|\bar{x}|$, we have

$$
M \models \varphi(\bar{a}) \quad \text { if and only if } \quad N \models \varphi(f(\bar{a})) .
$$

(iii) If $M$ is a substructure of $N$ and for every $\varphi(\bar{x}) \in \Phi$ and every $\bar{a} \in M$ with $|\bar{a}|=|\bar{x}|$, $M \models \varphi(\bar{a})$ if and only if $N \models \varphi(\bar{a})$, then we say that $M$ is a $\Phi$-elementary substructure of $N$ and that $N$ is a $\Phi$-elementary extension of $M$, denoted $M \preccurlyeq \Phi N$. As usual we may write $\preccurlyeq$ instead of $\preccurlyeq L$.

In the next section we will use the following result which is proved in the same way as the well-known Tarski-Vaught test [20]; for the proof we only need to observe that $L^{n}$ is closed under subformulas.

Fact 2.2. (Tarski-Vaught test for $L^{n}$ ) Suppose that $n$ is greater than the arity of every function symbol in the vocabulary of $L$ and let $M$ be an L-structure. For any subset $N$ of $M$, we have $N \preccurlyeq L^{n} M$ if and only if for every $\varphi(y, \bar{x}) \in L^{n}$ and every $\bar{a} \in N$ (with $|\bar{a}|=|\bar{x}|)$, if $M \models \exists y \varphi(y, \bar{a})$ then there is $b \in N$ such that $M \models \varphi(b, \bar{a})$.

Assumption 2.3. For the rest of this section we assume the following:

(1) $\Phi \subseteq L$ and $\Phi$ is closed under subformulas.

(2) $T \subseteq \Phi$ is a set of sentences.

(3) For every $M \models T$ a closure map $\mathrm{cl}_{M}$ is fixed such that if $M, N \models T, a_{i} \in M$, $b_{i} \in N$, for $i<\lambda$, and

$$
\left(M,\left(a_{i}: i<\lambda\right)\right) \equiv_{\Phi}\left(N,\left(b_{i}: i<\lambda\right)\right)
$$

then $\left\{a_{i}: i<\lambda\right\}$ is closed (with respect to $\mathrm{cl}_{M}$ ) if and only if $\left\{b_{i}: i<\lambda\right\}$ is closed (with respect to $\mathrm{cl}_{N}$ ). Because of this assumption we can, for simplicity of notation, omit the subscript ' $M$ ' in $\mathrm{cl}_{M}$ in the situations where we deal with a closure map.

(4) $\mathrm{cl}$ is uniformly locally finite with respect to $T$; that is, for every $m<\omega$ there is $m^{\prime}<\omega$ such that if $M=T, A \subseteq M$ and $|A| \leq m$, then $|\operatorname{cl}(A)| \leq m^{\prime}$. 
Remark 2.4. Natural examples of $\Phi$ which are closed under subformulas are $\Phi=L^{n}$ and

$$
\Phi=\{\varphi \in L: \text { the quantifier rank of } \varphi \text { is at most } n\} .
$$

See [20] for a definition of quantifier rank.

Definition 2.5. A non-empty class $\mathcal{A}$ of $L$-structures is called a $\Phi$-amalgamation class for $T$ if:

(1) Every structure in $\mathcal{A}$ is a model of $T$.

(2) $\mathcal{A}$ is closed under isomorphism.

(3) $\mathcal{A}$ is closed under $\Phi$-elementary substructures, i.e. if $N \in \mathcal{A}$ and $M \preccurlyeq_{\Phi} N$ then $M \in \mathcal{A}$.

(4) Whenever $M_{1}, M_{2} \in \mathcal{A}$ and $\bar{a} \in M_{1}, \bar{b} \in M_{2}$ are closed sequences of the same length and

$$
\left(M_{1}, \bar{a}\right) \equiv_{\Phi}\left(M_{2}, \bar{b}\right),
$$

then there are $N \in \mathcal{A}$ and a $\Phi$-elementary embedding $f: M_{2} \rightarrow N$ such that $M_{1} \preccurlyeq \Phi N$ and $f(\bar{b})=\bar{a}$.

Remark 2.6. If there is a $\Phi$-amalgamation class for $T$ then there is, by the downward Löwenheim-Skolem theorem, an amalgamation class for $T$ such that all structures in it are countable.

Examples 2.7. (a) Let $n \geq 4$, let $M$ be a tree such that for some $m<\omega$ no path in $M$ has length $m$ and let $T=T h_{L^{n}}(M)$. Moreover, for every $M \models T$ and every $A \subseteq M$ let $\operatorname{cl}(A)=A$. Then the class of all models of $T$ is an $L^{n}$-amalgamation class for $T$.

(b) Let $n \geq 3$, let $K$ be a finite field and let $T \subseteq L^{3}$ formalize the axioms of $K$-vector spaces in a language where scalar multiplication and vector addition are represented by function symbols. For every $M \models T$ and every $A \subseteq M$ let $\operatorname{cl}(A)$ be the substructure which is generated by $A$. Then the class of all models of $T$ is an $L^{n}$-amalgamation class for $T$. If we had defined $\mathrm{cl}$ as in (a) then $T$ would not have had any $L^{n}$-amalgamation class. This fact is a consequence of results in Section 3 and is discussed immediately after Theorem 3.7.

Definition 2.8. Suppose that $\mathcal{A}$ is a $\Phi$-amalgamation class for $T$.

(i) We say that $p \subseteq \Phi$ is a closed $(\Phi, \mathcal{A})$-type if there are $M \in \mathcal{A}$ and a closed finite sequence $\bar{a} \in M$ such that

$$
p=\{\varphi(\bar{x}) \in \Phi: M \models \varphi(\bar{a})\} .
$$

(ii) We say that an $L$-structure $M$ is $(\Phi, \omega, \mathcal{A})$-saturated for closed sets if whenever $\bar{b} \in N \in \mathcal{A}, \bar{a} \in M \cap N, \bar{a}$ and $\bar{b} \bar{a}$ are closed finite sequences and

$$
(M, \bar{a}) \equiv_{\Phi}(N, \bar{a}),
$$

then there exists $\bar{c} \in M$ such that $(M, \bar{a} \bar{c}) \equiv_{\Phi}(N, \bar{a} \bar{b})$, and hence, by Assumption $2.3(3), \bar{c} \bar{a}$ is closed.

Lemma 2.9. If $\mathcal{A}$ is a $\Phi$-amalgamation class for $T$ such that the set of all closed $(\Phi, \mathcal{A})$ types is countable, then there exists a countable L-structure $M$, such that

(i) $M \models T$

(ii) $M$ is $(\Phi, \omega, \mathcal{A})$-saturated, and

(iii) for every finite $\bar{a} \in M$ there exists $N \in \mathcal{A}$ such that $\bar{a} \in N$ and $(M, \bar{a}) \equiv_{\Phi}(N, \bar{a})$.

Proof. By Remark 2.6 we may assume that $\mathcal{A}$ is a $\Phi$-amalgamation class for $T$ which consists only of countable structures.

Then we use the idea in the proof of Fraïssé's theorem (see [20] for instance) to construct $M_{i} \in \mathcal{A}$, for $i<\omega$, such that

- $M_{i} \preccurlyeq{ }_{\Phi} M_{i+1}$, for all $i<\omega$, and 
- for any $i<\omega, \bar{a} \in M_{i}$ and $N \in \mathcal{A}$, if $\bar{a}, \bar{b} \in N, \bar{a}$ and $\bar{a} \bar{b}$ are closed and $\left(M_{i}, \bar{a}\right) \equiv_{\Phi}$ $(N, \bar{a})$, then there exists $j \geq i$ and $\bar{c} \in M_{j}$ such that $\left(M_{j}, \bar{a} \bar{c}\right) \equiv_{\Phi}(N, \bar{a} \bar{b})$.

Let $\pi: \omega^{3} \rightarrow \omega$ be a bijection such that $\pi(i, j, k) \geq i, j, k$ for all $i, j, k$ and let $p_{k}, k<\omega$, be an enumeration of all closed $(\Phi, \mathcal{A})$-types. Let $M_{0} \in \mathcal{A}$ be arbitrary. Now suppose that $M_{i}$ is defined for all $i<\ell+1, M_{i} \preccurlyeq \Phi M_{i+1}$ for all $i<\ell$ and that $\bar{a}_{i}^{j}, j<\omega$, is an enumeration of all closed finite sequences of elements from $M_{i}$, for $i<\ell+1$. Suppose that $\ell=\pi(i, j, k)$. If there exists $N \in \mathcal{A}$ and $\bar{b} \in N$ such that $\bar{a}_{i}^{j} \in N, \bar{a}_{i}^{j} \bar{b}$ is closed, $p_{k}=\left\{\varphi(\bar{x}, y) \in \Phi: N \models \varphi\left(\bar{a}_{i}^{j}, b\right)\right\}$ and $\left(M_{i}, \bar{a}_{i}^{j}\right) \equiv_{\Phi}\left(N, \bar{a}_{i}^{j}\right)$, then $\left(M_{\ell}, \bar{a}_{i}^{j}\right) \equiv_{\Phi}\left(N, \bar{a}_{i}^{j}\right)$ so, by condition (4) in the definition of a $\Phi$-amalgamation class, there are $M_{\ell+1} \in \mathcal{A}$ and $\bar{c} \in M_{\ell+1}$ such that $M_{\ell} \preccurlyeq \Phi M_{\ell+1}$ and $\left(M_{\ell+1}, \bar{a}_{i}^{j} \bar{c}\right) \equiv_{\Phi}\left(N, \bar{a}_{i}^{j} \bar{b}\right)$. Otherwise let $M_{\ell+1}=M_{\ell}$.

Let $M=\bigcup_{i<\omega} M_{i}$. Since $\Phi$ is closed under subformulas it follows from Lemma 2.11 below that $M_{i} \preccurlyeq \Phi M$, for all $i<\omega$, and from this we get (i). Conditions (ii) and (iii) follows from the construction of $M$.

Definition 2.10. If $M$ is a model as in Lemma 2.9 then we call $M$ a limit of $\mathcal{A}$.

Lemma 2.11. Suppose that $\Phi$ is closed under subformulas and that $M_{i} \preccurlyeq{ }_{\Phi} M_{i+1}$ for $i<\kappa$. If $M=\bigcup_{i<\kappa} M_{i}$ then $M_{i} \preccurlyeq \Phi M$ for every $i<\kappa$.

Proof. By induction on the complexity of formulas in $\Phi$.

See [20] for a definition of an unnested formula.

Lemma 2.12. Suppose that every unnested atomic formula of $L$ is equivalent, modulo $T$, to a formula in $\Phi$. Let $\mathcal{A}$ be a $\Phi$-amalgamation class for $T$ and suppose that $M$ and $N$ are limits of $\mathcal{A}$. Then for all closed finite sequences $\bar{a} \in M$ and $\bar{b} \in N$ with $|\bar{a}|=|\bar{b}|$,

$$
\text { if } \quad(M, \bar{a}) \equiv_{\Phi}(N, \bar{b}) \quad \text { then } \quad(M, \bar{a}) \equiv_{L}(N, \bar{b}),
$$

and in fact there is an isomorphism from $M$ onto $N$ which sends $\bar{a}$ to $\bar{b}$.

Proofsketch. Using properties (ii) and (iii) of Lemma 2.9 one carries out a back and forth argument which shows that there is an isomorphism from $M$ to $N$ which sends $\bar{a}$ to $\bar{b}$.

Corollary 2.13. Suppose that every unnested atomic formula of $L$ is equivalent, modulo $T$, to a formula in $\Phi$. Then a limit of a $\Phi$-amalgamation class $\mathcal{A}$ for $T$ is unique up to isomorphism.

Note that if, for example, $\Phi=L^{n}, n \geq 2$, every relation symbol has arity at most $n$ and every function symbol has arity less than $n$, then every unnested atomic $L$-formula belongs $\Phi$, so the condition about $\Phi$ in Lemma 2.12 and in Corollary 2.13 is satisfied.

Definition 2.14. An $L$-structure $M$ is $\Phi$-determined if for any closed finite sequences $\bar{a}, \bar{b} \in M$,

$$
\text { if } \quad(M, \bar{a}) \equiv_{\Phi}(M, \bar{b}) \quad \text { then } \quad(M, \bar{a}) \equiv_{L}(M, \bar{b}) .
$$

Hence, if $\Phi$ and $T$ satisfies the assumptions of Lemma 2.12 and $\mathcal{A}$ is a $\Phi$-amalgamation class for $T$, then a limit of $\mathcal{A}$ exists, is unique up to isomorphism and is $\Phi$-determined. We also have a partial converse. To state it we need the following definition:

Definition 2.15. An $L$-structure $M$ is $\omega$-homogeneous if for all finite sequences $\bar{a}, \bar{b} \in M$ such that $(M, \bar{a}) \equiv_{L}(M, \bar{b})$ and for every $c \in M$ there is $d \in M$ such that $(M, \bar{a} c) \equiv_{L}$ $(M, \bar{b} d)$.

Lemma 2.16. If $M$ a model of $T$ which is $\Phi$-determined and either finite, or infinite and $\omega$-homogeneous, then there is a $\Phi$-amalgamation class for $T$. 
Proofsketch. Take as the $\Phi$-amalgamation class all $N$ which are isomorphic to some countable $N^{\prime} \preccurlyeq \Phi M$.

We say that an infinite $L$-structure $M$ is $\omega$-categorical if $T h_{L}(M)$ is $\omega$-categorical.

Remark 2.17. A basic fact is that every $\omega$-categorical structure is $\omega$-homogeneous. Also, a complete theory is $\omega$-categorical if and only if for every model $M$ of the theory and every $n<\omega, S_{n}^{L}(\emptyset, M)$ is finite and there are only finitely many formulas with at most $n$ free variables, up to equivalence modulo the theory. (This is the well-known "Ryll-Nardzewski theorem" [20]).

For the next proposition, recall that $\Phi_{\bar{x}}=\{\varphi(\bar{x}): \varphi(\bar{x}) \in \Phi\}$. By combining the previous lemmas we get the following.

Proposition 2.18. Suppose that every unnested atomic formula in $L$ is equivalent to a formula in $\Phi$ and that for any $\bar{x}, \Phi_{\bar{x}}$ is finite up to equivalence modulo $T$. Then the following are equivalent:

(i) There exists a $\Phi$-amalgamation class for $T$.

(ii) There exists $M \models T$ which is $\Phi$-determined, and if $M$ is infinite, then $M$ is $\omega$ categorical (because, by Assumption 2.3 (4), cl is uniformly locally finite on M).

\section{Stability}

Now we will consider 'stability' and see how imposing a stability condition on an $L^{n}$-theory $T$ makes the limit $M$ of every amalgamation class for $T \omega$-stable. This together with the $\omega$-categoricity of $M$ ensures that there are arbitrarily large finite substructures of $M$ which are models of $T$.

Assumption 3.1. In this section we assume the following:

(1) For every theory $T$ that we speak about there is a closure map cl on the models of $T$ which is uniformly locally finite with respect to $T$. (See Assumption 2.3 (4).)

(2) If $T$ is an $L^{n}$-theory, $M, N \models T, a_{i} \in M, b_{i} \in N$, for $i<\lambda$ and

$$
\left(M,\left(a_{i}: i<\lambda\right)\right) \equiv_{L^{n}}\left(N,\left(b_{i}: i<\lambda\right)\right)
$$

then $\left\{a_{i}: i<\lambda\right\}$ is closed if and only if $\left\{b_{i}: i<\lambda\right\}$ is closed.

\section{Definition 3.2.}

(i) Suppose that $\mathcal{A}$ is an $L^{n}$-amalgamation class (for $T$ ). We say that $\mathcal{A}$ is stable in $L^{n}$ if for every $\varphi(\bar{x}, \bar{y}) \in L^{n}$ there exists $k_{\varphi}<\omega$ such that there does not exist $M \in \mathcal{A}$ and $\bar{a}_{i}, \bar{b}_{i} \in M$, for $i<k_{\varphi}$, satisfying $M \models \varphi\left(\bar{a}_{i}, \bar{b}_{j}\right) \Longleftrightarrow i \leq j$.

(ii) We adopt the convention that every finite structure is stable.

(iii) An infinite $L$-structure $M$ is stable if for every $\varphi(\bar{x}, \bar{y}) \in L$, there exists $k_{\varphi}<\omega$ such that there do not exist $\bar{a}_{i}, \bar{b}_{i} \in M$, for $i<k_{\varphi}$, satisfying $M=\varphi\left(\bar{a}_{i}, \bar{b}_{j}\right) \Longleftrightarrow i \leq j$.

(iv) A formula $\varphi(\bar{x}, \bar{y})$ is unstable with respect to a theory $T$ if there exist $M \models T$ and $\bar{a}_{i}, \bar{b}_{i} \in M, i<\omega$, such that $M \models \varphi\left(\bar{a}_{i}, \bar{b}_{j}\right) \Longleftrightarrow i \leq j$; otherwise $\varphi(\bar{x}, \bar{y})$ is stable with respect to $T$.

Proposition 3.3. Suppose that $n$ is greater than the arity of every function symbol in the vocabulary of $L$ and that $n$ is at least as great as the arity of every relation symbol in the vocabulary of $L$. If $T$ is a complete $L^{n}$-theory such that $S_{n}^{n}(T)$ is finite then the following are equivalent:

(i) There is a stable $L^{n}$-amalgamation class for $T$.

(ii) $T$ has a stable model which is $L^{n}$-determined (and hence $\omega$-categorical if it is infnite). 
Proofsketch By Fact 1.5, the assumption that $S_{n}^{n}(T)$ is finite implies that there are only finitely many $L^{n}$-formulas up to equivalence modulo $T$ and hence for any sequence of variables $\bar{x}$ (of any length) $L_{\bar{x}}^{n}$ is finite up to equivalence modulo $T$.

Hence Proposition 2.18 gives all except the statement about stability. But one direction of this follows from the fact that

- in (ii) we take the model of $T$ to be the limit of a stable $L^{n}$-amalgamation class for $T$, and

- if the formulas $\varphi_{i}(\bar{x}, \bar{y}), i=1, \ldots, m$, are stable with respect to a complete $L$ theory, then every boolean combination of the $\varphi_{i}$ 's is stable with respect to the same complete $L$-theory. (This can be proved directly by using Ramsey's theorem, but it also follows from the basic work on stable formulas by Shelah [31].)

And conversely, given a model $M$ satisfying the conditions in (ii), a stable $L^{n}$-amalgamation class is obtained by taking all $L^{n}$-elementary substructures of $M$ (and structures isomorphic to these) as in Lemma 2.16 .

Definition 3.4. An $L$-structure $M$ is $\omega$-stable if whenever $M^{\prime} \equiv_{L} M, A \subseteq M^{\prime}$ and $|A| \leq \omega$ then $\left|S_{1}^{L}(A, M)\right| \leq \omega$.

A basic fact from stability theory is that if $M$ is $\omega$-stable then $M$ is stable. The next lemma, which tells that under certain circumstances the converse also holds, will be essential here.

Lemma 3.5. Suppose that $M$ is an infinite L-structure such that $\operatorname{cl}(A)=A$ for every $A \subseteq M$ and $S_{n}^{n}(\emptyset, M)$ is finite. If $M$ is stable and $L^{n}$-determined then $M$ is $\omega$-stable.

Proofsketch. Under the premises of the lemma it follows that any $L$-formula is equivalent, modulo $T h_{L}(M)$, to a boolean combination of $L^{n}$-formulas and there are only finitely many $L^{n}$-formulas up to equivalence modulo $T h_{L^{n}}(M)$. Thus for every complete $L$-type $p(\bar{x})$ over a set $A, p(\bar{x})$ is determined by $p(\bar{x}) \cap L^{n}(A)$. Now the lemma follows from the fact that if $0<m<\aleph_{0}$ then $\left(\aleph_{0}\right)^{m}=\aleph_{0}$ and from Shelah's "unstable formula theorem" ([31], Theorem II.2.2), which tells us that if $\varphi\left(v_{1}, \bar{y}\right)$ is stable and $A \subseteq M$ is countable then $S_{1}^{\left\{\varphi\left(v_{1}, \bar{y}\right)\right\}}(A)$ is countable.

Suppose that $n$ is greater than the arity of every function symbol in the vocabulary of $L$ and greater than or equal to the arity of every relation symbol in the vocabulary of $L$. From Proposition 3.3 and Lemma 3.5 it follows that if $T$ is a complete $L^{n}$-theory such that $S_{n}^{n}(T)$ is finite and there is a stable $L^{n}$-amalgamation class for $T$, then $T$ has a model which is $\omega$-stable and, if it is infinite, $\omega$-categorical.

Next we state the crucial result which will give us finite models for every complete $L^{n}$-theory $T$ such that $S_{n}^{n}(T)$ is finite and there is a stable $L^{n}$-amalgamation class for $T$.

Theorem 3.6. (Cherlin, Harrington, Lachlan [4]) If $M$ is $\omega$-categorical and $\omega$-stable, $M \models$ $\varphi$ and $A$ is a finite subset of $M$, then there exists a finite substructure $N \subseteq M$ such that $N \models \varphi$ and $A \subseteq N$.

Recall that, by Fact 1.6, if $T$ is a complete $L^{n}$-theory such that $S_{n}^{n}(T)$ is finite then $T$ is axiomatized by an $L^{n}$-sentence. Thus, applying Theorem 3.6 and previous results we get:

Theorem 3.7. Suppose that $n$ is greater than the arity of every function symbol in the vocabulary of $L$ and greater or equal to the arity of every relation symbol in the vocabulary of $L$. If $T$ is a complete $L^{n}$-theory such that $S_{n}^{n}(T)$ is finite then the following hold:

(i) If $M$ is a model of $T$ which is $\omega$-categorical and $\omega$-stable, then for any finite $A \subset M$ there is a finite $N \preccurlyeq L^{n} M$ such that $A \subseteq N$, and consequently $N \models T$.

(ii) Suppose that for every $M \models T$ and $A \subseteq M, \operatorname{cl}(A)=A$. If there is a stable $L^{n}$ amalgamation class $\mathcal{A}$ for $T$ such that $\mathcal{A}$ contains at least one infinite structure then $T$ has arbitrarily large finite models. 
Observe the assumption in part (ii) of the above theorem that the closure operation is trivial. We now turn to the case when 'cl' is not trivial (i.e. for some $A, \operatorname{cl}(A) \neq A)$. An example of such a situation is if $M$ is an infinite vector space over a finite field and $T=T h_{L^{n}}(M)$, for sufficiently large $n$. Then there cannot exist a trivial closure operation cl and a stable $L^{n}$-amalgamation class for $T$, with respect to this closure operation, which contains an infinite structure. The reason is that it would imply the existence of an infinite vector space, over the same field, which is $L^{n}$-determined and this is impossible. For in every infinite model of $T$ we can choose $m$ greater than $n$ and on the one hand a linearly independent sequence $\bar{a}=\left(a_{1}, \ldots, a_{m}\right)$ and on the other hand a sequence $\bar{b}=$ $\left(b_{1}, \ldots, b_{m}\right)$ such that $\bar{b}$ is not linearly independent but every proper subtuple of $\bar{b}$ is linearly independent. With this choice, $\bar{a}$ and $\bar{b}$ have the same $L^{n}$-type but not the same $L$-type, so the structure is not $L^{n}$-determined.

Motivated by this example we would like to find some amalgamation property for complete $L^{n}$-theories $T$ which holds also for the example of vector spaces and which implies the existence of an $\omega$-categorical and $\omega$-stable model of $T$, so that we are in position to apply Theorem 3.6.

Definition 3.8. Let $T$ be an $L^{n}$-theory. $T$ has the strong $L^{n}$-amalgamation property over countable models if, whenever

$M_{0}=T, M_{0} \preccurlyeq L^{n} M_{i}$, where $M_{i}$ is countable for $i=1,2, \bar{a}_{1} \in M_{1}, \bar{a}_{2} \in M_{2}$ are finite sequences and $\left(M_{1}, \operatorname{cl}\left(\bar{a}_{1}\right) M_{0}\right) \equiv_{L^{n}}\left(M_{2}, \operatorname{cl}\left(\bar{a}_{2}\right) M_{0}\right)$

then

there are $M$ and $L^{n}$-elementary embeddings $f_{i}: M_{i} \rightarrow M, i=1,2$, such that $f_{1}\left(\bar{a}_{1}\right)=f_{2}\left(\bar{a}_{2}\right)$ and $f_{i}$ is the identity on $M_{0}$ for $i=1,2$.

Remark 3.9. Suppose that $M$ is a vector space over a finite field and $T=T h_{L^{n}}(M)$, for $n$ larger than the number of elements in the field. Using the elementary theory of vector spaces it is now easy to verify that $T$ has the strong $L^{n}$-amalgamation property over countable models. Also one can easily verify that if $\mathrm{cl}$ is taken to be linear closure then there is a stable $L^{n}$-amalgamation class for $T$ with respect to this closure operation.

Theorem 3.10. (Baldwin, Lessmann [2]) Suppose that $T$ is a complete $L^{n}$-theory such that $S_{n}^{n}(T)$ is finite and $T$ has the strong $L^{n}$-amalgamation property over countable models.

(i) If $M \models T$ and $M$ is stable and $L^{n}$-determined then $M$ is $\omega$-stable.

(ii) If there is a stable $L^{n}$-amalgamation class $\mathcal{A}$ for $T$ such that $\mathcal{A}$ contains at least one infinite structure then $T$ has arbitrarily large finite models; these can be taken as $L^{n}$-elementary substructures of the limit of $\mathcal{A}$.

Proofsketch. (ii) follows from (i) and earlier results. Concerning (i): The assumptions that $S_{n}^{n}(T)$ is finite, $M$ is stable and $L^{n}$-determined (so $M$ is $\omega$-categorical) imply, via Shelah's "unstable formula theorem" [31], that for any countable $A \subseteq M^{\prime} \equiv_{L} M, S_{1}^{L^{n}}\left(A, M^{\prime}\right)$ is countable. The useful consequence of the strong $L^{n}$-amalgamation property over countable models is that if $N \preccurlyeq N^{\prime} \models T$, where $N$ is countable, and $\bar{a}, \bar{b} \in N^{\prime}$ are finite sequences then

$$
\left(N^{\prime}, \operatorname{cl}(\bar{a}) N\right) \equiv L^{n}\left(N^{\prime}, \operatorname{cl}(\bar{b}) N\right)
$$

implies

$$
\left(N^{\prime}, \operatorname{cl}(\bar{a} N)\right) \equiv_{L^{n}}\left(N^{\prime}, \operatorname{cl}(\bar{b} N)\right) .
$$

This property together with the assumption that $M$ is $L^{n}$-determined (which by the $\omega$ categoricity of $M$ implies that any $N \equiv_{L} M$ is $L^{n}$-determined) shows that $M$ is $\omega$-stable, by a counting types argument. 


\section{ReCuRsive Bounds}

In this section we derive results about recursive upper bounds of the size of the least model of $L^{n}$-theories.

We will use the theory of smoothly approximable structures which is presented in detail in [3]. Every structure which is $\omega$-categorical and $\omega$-stable is smoothly approximable which essentially follows from [4], but also see [24]. One of several equivalent ways of defining 'smoothly approximable' is the following:

Definition 4.1. An $L$-structure $M$ is smoothly approximable if $M$ is $\omega$-categorical and if every $L$-sentence which is true in $M$ is true in a finite substructure $N \subseteq M$ such that

(1) for every $\theta(\bar{x}) \in L$ there is $\chi(\bar{x}) \in L$ such that

$$
\{\bar{a} \in N: M \models \theta(\bar{a})\}=\{\bar{a} \in N: N \models \chi(\bar{a})\} \text {, and }
$$

(2) for all $\bar{a}, \bar{b} \in N$ of the same finite length

$$
(N, \bar{a}) \equiv_{L}(N, \bar{b}) \Longleftrightarrow(M, \bar{a}) \equiv_{L}(M, \bar{b}) .
$$

We derive our results from the following theorem which does not directly speak about smoothly approximable structures.

Theorem 4.2. (Cherlin, Hrushovski [3]) We can effectively decide for a given sentence and $k<\omega$ if that sentence has a finite model $M$ such that $\left|S_{4}^{L}(\emptyset, M)\right|=k$.

For our purposes we now define a recursive function $\mathfrak{f}: \omega^{2} \rightarrow \omega$ as follows:

Let $\mathfrak{f}(n, k)=k$ if $n<2$ or $k=0$.

Now suppose that $n \geq 2$ and $k \geq 1$.

- Let $\varphi_{1}, \ldots, \varphi_{m}$ be an enumeration of all sentences (up to equivalence) of quantifier rank at most $k+n$ in a language $\mathcal{L}_{k}$ such that for every $1 \leq i \leq n$ the vocabulary of $\mathcal{L}_{k}$ contains exactly $k$ constant symbols and exactly $k i$-ary relation symbols, but no function symbols, and we assume that ' $=$ ' is one of the binary relation symbols.

- For $1 \leq i \leq m$, let $L_{i}$ be the language built up from the constant symbols and the relation symbols that occur in $\varphi_{i}$ and the identitity symbol ' $=$ ' (but no other symbols from the vocabulary of $\mathcal{L}_{k}$ ). Then use Theorem 4.2 to decide if $\varphi_{i}$ has a finite model $M_{i}$ such that $\left|S_{4}^{L_{i}}\left(\emptyset, M_{i}\right)\right| \leq k$;

- if such a model of $\varphi_{i}$ exists then search until we find such $M_{i}$ and let $\ell_{i}=\left|M_{i}\right|$; otherwise let $\ell_{i}=0$.

- Then let $\mathfrak{f}(n, k)=\max \left\{\ell_{1}, \ldots, \ell_{m}\right\}$.

If we had allowed function symbols in the language $\mathcal{L}_{k}$ appearing in the definition of $\mathfrak{f}$, then there would have been infinitely many formulas of rank at most $n \leq k+n$ (even quantifier free formulas) that are non-equivalent. When computing $\mathfrak{f}$ we depend on the fact that (with the stated definition) there are only finitely many non-equivalent $\mathcal{L}_{k}$-formulas with quantifier rank at most $k+n$.

Definition 4.3. Let $T$ be a complete $L^{n}$-theory. As in the previous sections we associate a uniformly locally finite closure operation $\mathrm{cl}$ with models of $T$. We now define a function $\mathrm{cl}^{*}: \omega \rightarrow \omega$ as follows:

$$
\operatorname{cl}^{*}(n)=\max \{|\operatorname{cl}(A)|: A \subseteq M \models T,|A| \leq n\} .
$$

Corollary 4.4. Let $T$ be a complete $L^{n}$-theory such that $S_{n}^{n}(T)$ is finite and let $\mathrm{cl}$ be the closure operation associated with models of $T$. Also assume that $n \geq \mathrm{cl}^{*}(4)$ and that the vocabulary of $L$ contains no function symbols and that the arity of every relation symbol is at most $n$. If $M=T$, where $M$ is smoothly approximable and $\left|S_{\mathrm{cl}^{*}(4)}^{n}(\emptyset, M)\right|=\left|S_{\mathrm{cl}^{*}(4)}(\emptyset, M)\right|$, then $\varphi$ has a finite model of cardinality at most $\mathfrak{f}\left(n,\left|S_{n}^{n}(\emptyset, M)\right|\right.$ ) (where $S_{n}^{n}(\emptyset, M)=S_{n}^{n}(T)$ ). 
Proof. Suppose that $T, L, n$ and $M$ satisfies the premises of the corollary. By renaming symbols if necessary we may assume, without loss of generality, that $L \subseteq \mathcal{L}_{k}$ where $\mathcal{L}_{k}$ is the language that occurs in the definition of $\mathfrak{f}$, with $k=\left|S_{n}^{n}(T)\right|$. By Fact 1.6, $T$ is axiomatized by an $L^{n}$-sentence with quantifier rank at most $\left|S_{n}^{n}(T)\right|+n=k+n$. Since $M$ is smoothly approximable it follows that $M$ has a finite substructure $N$ such that $N=T$ and $\left|S_{4}(\emptyset, N)\right| \leq\left|S_{4}(\emptyset, M)\right|$. From the assumptions that $n \geq \operatorname{cl}^{*}(4)$ and $\left|S_{\mathrm{cl}^{*}(4)}^{n}(\emptyset, M)\right|=\left|S_{\mathrm{cl}^{*}(4)}(\emptyset, M)\right|$ it follows that

$$
\left|S_{4}(\emptyset, N)\right| \leq\left|S_{4}(\emptyset, M)\right| \leq\left|S_{\mathrm{cl}^{*}(4)}(\emptyset, M)\right|=\left|S_{\mathrm{cl}^{*}(4)}^{n}(\emptyset, M)\right| \leq\left|S_{n}^{n}(\emptyset, M)\right|=k .
$$

By the definition of $\mathfrak{f}$, there is a model of $T$ with cardinality at most $\mathfrak{f}\left(n,\left|S_{n}^{n}(\emptyset, M)\right|\right)$.

Corollary 4.5. Let $n \geq 4$ and let $L$ be a language with finite vocabulary which contains no function symbols and in which all relation symbols have arity at most $n$. If $T$ is a complete $L^{n}$-theory such that

- $S_{n}^{n}(T)$ is finite,

- $n \geq \operatorname{cl}^{*}(4)$,

- for every $M \models T$ and every $A \subseteq M, \operatorname{cl}(A)=A$, or $T$ has the strong $L^{n}$ amalgamation property over countable models, and

- there is a stable $L^{n}$-amalgamation class for $T$ (with respect to $\mathrm{cl}$ ),

then $T$ has a model of cardinality at most $\mathfrak{f}\left(n,\left|S_{n}^{n}(T)\right|\right)$.

Proof. Suppose that $T$ satisfies the above conditions. First note that (by Fact 1.6) $T$ is axiomatized by an $L^{n}$-sentence with quantifier rank $\leq\left|S_{n}^{n}(T)\right|+n$. By results in the previous section, $T$ has an $\omega$-categorical and $\omega$-stable (hence smoothly approximable) model $M$ which is $L^{n}$-determined (with respect to the given closure operator). Then $\left|S_{\mathrm{cl}^{*}(4)}^{n}(\emptyset, M)\right|=\left|S_{\mathrm{cl}^{*}(4)}(\emptyset, M)\right|$, so by Corollary $4.4, T$ has a model $N$ with cardinality at most $\mathfrak{f}\left(n,\left|S_{n}^{n}(\emptyset, M)\right|\right)=\mathfrak{f}\left(n,\left|S_{n}^{n}(T)\right|\right)$.

Remark 4.6. Grohe [17] has shown that if $n \geq 3$ then there does not exist a recursive function $f_{n}: \omega \rightarrow \omega$ such that for every complete $L^{n}$-theory $T$ with finite models,

$$
\min \{|M|: M \models T\} \leq f_{n}\left(\left|S_{n}^{n}(T)\right|\right) .
$$

Except for the results presented here, an existence result about recursive upper bounds has also been obtained by Dawar in [5]. The hypothesis of Dawar's result is that the class $\mathcal{C}$ of finite structures considered (where $\mathcal{C}$ could be the class of all finite models of an $L^{n}$-theory, for example) has the 'weak n-Ehrenfeucht-Mostowski property'. Roughly speaking, this property says that every sufficiently long " $L^{n}$-indiscernible" sequence in a structure in $\mathcal{C}$ can both be extended in some $L^{n}$-elementary extension which belongs to $\mathcal{C}$ and reduced (as long as it does not become too short) in some $L^{n}$-elementary substructure that belongs to $\mathcal{C}$.

The question arises: How general can a class, $\mathcal{T}$, of complete $L^{n}$-theories with finite models be if we require that there exists a recursive function $f$ such that $\min \{|M|: M=$ $T\} \leq f\left(\left|S_{n}^{n}(T)\right|\right)$ for all $T \in \mathcal{T}$ ? Another problem is to determine such a function $f$ more precisely (polynomial, exponential, etc.), perhaps starting with some smaller class of theories over which we have more control.

\section{Simple, possibly not smoothly approximable structures}

This paper has focused on obtaining finite models for a complete $L^{n}$-theory $T$ by showing that $T$ has an infinite model $M$ which has the finite submodel property, by which we mean that every sentence which is true in $M$ is true in a finite substructure of $M$. As stated in Theorem 3.6, every $\omega$-categorical $\omega$-stable structure has the finite submodel property. The same holds for the more inclusive class of smoothly approximable structures which also contains unstable examples (see [3]). 
There are natural examples of structures which have the finite submodel property but are not smoothly approximable, such as the random (bipartite) graph $G_{r g}$ [24]. $G_{r g}$ can be defined as the Fraïssé limit of the class of all finite graphs, or alternatively one can give an explicit axiomatization of the complete theory of $G_{r g}$; see for instance $[12,20]$ for more about the random graph. (The random bipartite graph is obtained similarly by considering the class of all finite graphs expanded with an equivalence relation with exactly two classes subject to the condition that edges may only occur between elements in different classes.) The random (bipartite) graph has the following model theoretical properties: it is $\omega$-categorical with elimination of quantifiers, (super)simple (but unstable) with SU-rank 1 and has trivial forking; see for instance [20] and [32] for these model theoretic and stability/simplicity theoretic notions. The fact that $G_{r g}$ has SU-rank 1 implies that the algebraic closure operation 'acl' forms a pregeometry on $G_{r g}$ (see [20]).

Before continuing we note that there is a line of research $[28,13,14,15]$, not discussed here, which studies the connection between classes of finite structures in which definable sets have uniform behaviour, asymptotically, and (infinite) simple structures with finite SUrank and with a measure on the definable subsets, but which are not necessarily smoothly approximable. A question not answered here is whether the approach in this article has anything in common with the work about 'asymptotic classes' and 'measurable structures' (the random graph fits within both frameworks).

Work in two different directions has been carried out by the author to prove the finite submodel property for classes of structures which contain the random (bipartite) graph. One direction of research [10] studies $\omega$-categorical structures on which the algebraic closure operation forms a pregeometry. The other direction of research [11, 26] studies structures which are $\omega$-categorical, simple with finite SU-rank and have trivial forking. In both directions a probabilistic argument is involved in proving the finite submodel property. In order to carry out this argument we need to assume that definable relations are "sufficiently independent" from each other in senses that are made precise in [10] and [26]. It seems that without any assumption about "sufficient independence" we are in a difficult situation with respect to proving or disproving the finite submodel property. We say more about this in the last paragraph of Section 6.

The notion of "sufficient independence" which is considered in [26] is called the " $n$ embedding of types property' (for a natural number $n \geq 2$ ), with respect to certain kinds of "generators". Before stating the main result of [26] we introduce some notation from stability/simplicity theory and explain, rougly, the involved notions. We assume familiarity with imaginary elements (see [20] or [32] for example). By $A \underset{C}{\downarrow} B$ we mean that $A$ is independent from $B$ over $C$ (see for example [32] for a definition of 'independence'). The negation of $A \underset{C}{\downarrow_{B}} B$ is denoted by $A \underset{C}{\chi_{C}} B$. A complete first-order theory $T$ has trivial dependence (or forking) if whenever $A, B_{1}, B_{2}, C$ are subsets of $M^{\text {eq }}$ where $M \models T$ and $A \underset{C}{\chi_{C}}\left(B_{1} \cup B_{2}\right)$, then $A \underset{C}{\chi_{i}} B_{i}$ for $i=1$ or $i=2$.

Here is a rough description of the $n$-embedding of types property (with respect to all/simple generators). Suppose that $T$ is a complete first-order theory which is simple (see [32] for a definition of 'simple') and assume that $M=T$. As usual, $M^{\text {eq }}$ denotes the extension of $M$ by imaginary elements and 'algebraic closure' is taken in the structure $M^{\text {eq }}$ (see [20] for a definition of algebraic closure). We identify every natural number $n$ with the set $\{0, \ldots, n-1\}$ and let $\mathcal{P}(n)$ be the set of all subsets of $n$ and $\mathcal{P}^{-}(n)=\mathcal{P}(n)-\{n\}$. Suppose that $A_{i}^{0}$ and $B_{i}^{0}$, for $i \in n$, are subsets of $M^{\mathrm{eq}}$ and that, for every $w \in \mathcal{P}^{-}(n), A_{w}$ is the algebraic closure of $\bigcup_{i \in w} A_{i}^{0}$ and $B_{w}$ is the algebraic closure of $\bigcup_{i \in w} B_{i}^{0}$. Also assume that

- for all $w, w^{\prime} \in \mathcal{P}^{-}(n), A_{w} \underset{A_{w \cap w^{\prime}}}{\perp} A_{w^{\prime}}$ and $B_{w} \underset{B_{w \cap w^{\prime}}}{\perp} B_{w^{\prime}}$, and 
- for every $w \in \mathcal{P}^{-}(n)$ there is an elementary map (see [20]) $f_{w}$ from $A_{w}$ onto $B_{w}$ such that $f_{w}\left(A_{i}^{0}\right)=B_{i}^{0}$ for every $i \in w$, and if $w \subseteq w^{\prime}$ then $f_{w^{\prime}}$ extends $f_{w} \backslash \bigcup_{i \in w} A_{i}^{0}$. The $n$-embedding of types property says (omitting some details) that if $\bar{a}=\left(a_{1}, \ldots, a_{r}\right)$ is a sequence of elements from $M^{\mathrm{eq}}$ which does not contain any element from the algebraic closure of $\bigcup_{w \in \mathcal{P}^{-}(n)} A_{w}$, then there are $\bar{b}=\left(b_{1}, \ldots, b_{r}\right)$ in $M^{\text {eq }}$ (we can assume that $M$ is sufficiently saturated) and, for $w \in \mathcal{P}^{-}(n)$, elementary maps $g_{w}: A_{w} \cup\left\{a_{1}, \ldots, a_{r}\right\} \rightarrow$ $B_{w} \cup\left\{b_{1}, \ldots, b_{r}\right\}$ such that $g_{w} \bigcup_{i \in w} A_{i}^{0}=f_{w} 川 \bigcup_{i \in w} A_{i}^{0}$ and if $w \subseteq w^{\prime}$ then $g_{w^{\prime}}$ extends $g_{w} \backslash \bigcup_{i \in w} A_{i}^{0}$.

The phrase 'with respect to all/simple generators' when stating the condition ' $n$-embedding of types property with respect to all/simple generators' in the next theorem refers to the conditions (if any) that we impose on the sets $A_{i}^{0}, B_{i}^{0}$, for $i \in n$. In [26] the sets $A_{i}^{0}, B_{i}^{0}$, $i \in n$, are called the "generators" of the sets $A_{m}, B_{m}, m \in \mathcal{P}^{-}(n)$. Every stable theory (see $[20,31,32]$ for the notion 'stable') has the $n$-embedding of types property with respect to simple generators for every $1<n<\omega[26]$. The complete theory of the random graph [20] has the $n$-embedding of types property with respect to all generators for every $1<n<\omega$ [26].

Theorem 5.1. [26] Suppose that there is $m<\omega$ such that every function symbol of the language of $M$ has arity at most $m$. If Th $(M)$ is $\omega$-categorical, simple with finite SU-rank, has trivial dependence and, for every $1<k<\omega$, has the $k$-embedding of types property with respect to all generators, then $M$ has the finite submodel property. If the SU-rank of $T h(M)$ is 1, then the phrase 'with respect to all generators' can be replaced by the phrase 'with respect to simple generators', a weaker hypothesis.

It follows from Lemma 5.3, below, that Theorem 5.1 holds also if we replace 'trivial dependence' with ' $n$-degenerate dependence for some $n<\omega$ ', where $n$-degenerate dependence is defined as follows.

Definition 5.2. Let $T$ be a complete simple $(L-)$ theory. We say that $T$ has $n$-degenerate dependence if the following holds: Whenever $M \models T$ and $A, B, C \subseteq M$ and $A \underset{C}{\swarrow_{C}} B$ then there is $B^{\prime} \subseteq B$ such that $\left|B^{\prime}\right| \leq n$ and $A \underset{C}{\not} B^{\prime}$.

Observe that trivial dependence implies 1-degenerate dependence.

Lemma 5.3. Suppose that $T$ is $\omega$-categorical, simple with finite SU-rank and with $n$ degenerate dependence for some $n<\omega$. Then $T$ has trivial dependence.

Proof sketch. Suppose that $T$ satisfies the premises of the lemma. By Corollary 4.7 in [18] and Lemma 3.22 in [7], it is sufficient to show that every type with SU-rank 1 is trivial, i.e. if $D$ is the set of realizations of the type, in $M^{\text {eq }}$ where $M$ is a sufficiently saturated model of $T$, then the restriction to $D$ of the algebraic closure operator forms a trivial pregeometry. For a contradiction, suppose that there is a nontrivial type of SU-rank 1. By Corollary 3.17 in [7] there is a definable subset of $\mathcal{M}^{e q}$ on which the algebraic closure is a projective geometry over a finite field. Now a contradiction can be derived in the same way as in the last two paragraphs of the proof of Proposition 8.7 in [8].

Now we can derive a corollary which applies to $L^{n}$-theories and amalgamation classes. Suppose that $\Phi$ is a subset of $L$ and $T$ is a set of sentences from $\Phi$ such that $\Phi$ is closed under subformulas, every unnested atomic formula of $L$ is equivalent, modulo $T$, to a formula in $\Phi$, for every $\bar{x}, \Phi_{\bar{x}}$ is finite up to equivalence modulo $T$. Also suppose that $\mathcal{A}$ is a $\Phi$-amalgamation class for $T$. Note that the assumptions about $\Phi$ hold if $\Phi=L^{n}$ and $n$ is greater than the arity of every symbol in the vocabulary. Then the assumptions of Lemma 2.9 and of Corollary 2.13 are satisfied, so by these results a unique limit of $\mathcal{A}$ exists. By Lemma 2.12 and Proposition 2.18 the limit of $\mathcal{A}$ is finite or $\omega$-categorical. 
Corollary 5.4. Assume that there is $m<\omega$ such that every function symbol has arity at most $m$. Let $T$ be a set of sentences from $\Phi \subseteq L$ where $\Phi$ is closed under subformulas. Suppose that, for every $\bar{x}, \Phi_{\bar{x}}$ is finite up to equivalence modulo $T$ and that every unnested atomic formula is equivalent to a formula in $\Phi$, modulo $T$. Moreover, suppose that $T$ has a $\Phi$-amalgamation class $\mathcal{A}$ with a limit $M$ such that $M$ is simple with finite $S U$-rank and has both $n$-degenerate forking for some $n<\omega$ and the $k$-embedding of types property with respect to all generators for every $1<k<\omega$. Then $T$ has arbitrarily large finite models (which can be taken as substructures of $M$ ).

The following example illustrates the notions and assumptions of the previous corollary.

Example 5.5. Let the vocabulary of the language $L$ be $\{=, E\}$ where $E$ is a binary relation symbol. Let $\chi$ be the sentence $\forall x_{1}, x_{2}\left(\neg E\left(x_{1}, x_{1}\right) \wedge\left(E\left(x_{1}, x_{2}\right) \rightarrow E\left(x_{2}, x_{1}\right)\right)\right)$. For every $n \geq 2$ and every $w \subseteq\{1, \ldots, n-1\}$ let $\theta_{w}^{n}\left(x_{1}, \ldots, x_{n}\right)$ be the formula

$$
\bigwedge_{i \in w} E\left(x_{i}, x_{n}\right) \wedge \bigwedge_{i \notin w} \neg E\left(x_{i}, x_{n}\right)
$$

and let $\varphi_{w}^{n}$ be the sentence

$$
\forall x_{1}, \ldots, x_{n-1}\left(\bigwedge_{i \neq j} x_{i} \neq x_{j} \rightarrow \exists x_{n} \theta_{w}^{n}\left(x_{1}, \ldots, x_{n}\right)\right) .
$$

Then let $\varphi^{n}$ be the conjunction of $\chi$ and every $\varphi_{w}^{n}$ as $w$ ranges over subsets of $\{1, \ldots, n-1\}$, so $\varphi^{n} \in L^{n}$. Also, every model of $\varphi^{n}$ is an undirected graph, or just 'graph' for brevity.

Fix an arbitrary natural number $n \geq 2$. Recall that, by Definition 2.1, $M \equiv_{L^{n}} N$ means that $M$ and $N$ are $L^{n}$-elementarily equivalent, i.e. satisfy exactly the same $L^{n}$-sentences, From the $n$-pebble game characterization of $L^{n}$-elementary equivalence [23, 29] it follows that if $M$ and $N$ are models of $\varphi^{n}$, then Duplicator (or "player II" or " $\exists "$ ") has a winning strategy in the $n$-pebble game on $M$ and $N$ in $\omega$ rounds, and therefore $M \equiv_{L^{n}} N$. It follows that $T^{n}=\left\{\varphi^{n}\right\}$ is a complete $L^{n}$-theory (in the sense of Definition 1.1 (vii)).

Infinite models of $\varphi^{n}$ exist since for every sequence $a_{1}, \ldots, a_{n-1}$ from a graph and every $w \subseteq\{1, \ldots, n-1\}$ we can add a new element $b$ to the graph and extend the interpretetation of $E$ so that $E\left(a_{i}, b\right)$ holds if and only if $i \in w$. By repeating this process systematically in $\omega$ steps (if we start with a finite structure) we can make sure that the union of the graphs created in the process is a model of $\varphi^{n}$. In fact, by reasoning similarly as has been outlined, we can show the following.

(I) For every graph $G$ (finite or infinite) there is a graph $M \models \varphi^{n}$ such that $G$ is a substructure of $M$.

From the $n$-pebble game characterization of ' $M \equiv_{L^{n}} N^{\prime}$ ' we can also derive the the following.

(II) If $M$ and $N$ are models of $\varphi^{n}, m<\omega, a_{1}, \ldots, a_{m} \in M$ are different elements and $b_{1}, \ldots, b_{m} \in N$ are different elements, then $\left(M, a_{1}, \ldots, a_{m}\right) \equiv_{L^{n}}\left(N, b_{1}, \ldots, b_{m}\right)$ if and only if for all $i, j \in\{1, \ldots, m\}, M \models E\left(a_{i}, a_{j}\right) \Longleftrightarrow N \models E\left(b_{i}, b_{j}\right)$

Consequently, if $M, N \models \varphi^{n}$ then every embedding $f: M \rightarrow N$ is an $L^{n}$-elementary embedding. In particular, if $M, N \models \varphi^{n}$ then $M \preccurlyeq L^{n} N$ if and only if $M$ is a substructure of $N$.

For every $M=\varphi^{n}$ and every $A \subseteq M$, define $\operatorname{cl}(A)=A$, so every subset of every model of $\varphi^{n}$ is closed. Let $\mathcal{A}$ be the class of all models of $T^{n}=\left\{\varphi^{n}\right\}$. From the definition of $\mathcal{A}$ it immediately follows that (1)-(3) in the definition of an $L^{n}$-amalgamation class for $T^{n}$ (Definition 2.5) are satisfied. We verify that also (4) in the same definition holds. Suppose that $M_{1}, M_{2} \in \mathcal{A}, a_{1}, \ldots, a_{m} \in M_{1}, b_{1}, \ldots, b_{m} \in M_{2}$ and that

$$
\left(M_{1}, a_{1}, \ldots, a_{m}\right) \equiv_{L^{n}}\left(M_{2}, b_{1}, \ldots, b_{m}\right) .
$$


To simplify the argument, without loss of generality, we may assume that $a_{i}=b_{i}$ for $i=1, \ldots, m$, and that $M_{1} \cap M_{2}=\left\{a_{1}, \ldots, a_{m}\right\}$. By (I), the graph $M_{1} \cup M_{1}$ (where $M_{1} \cup M_{2} \models E(a, b) \Longleftrightarrow M_{1}=E(a, b)$ or $\left.M_{2} \models E(a, b)\right)$ is a substructure of some model $N$ of $T^{n}=\left\{\varphi^{n}\right\}$. Since $M_{1}, M_{2} \models \varphi^{n}$, it follows from (II) that $M_{i} \preccurlyeq L^{n} N$ for $i=1,2$. Hence $\mathcal{A}$ is an $L^{n}$-amalgamation class for $T^{n}$.

By Lemma 2.9 and Corollary $2.13, \mathcal{A}$ has a unique limit $M$ which is countable (by the definition of limit). By Lemma 2.12, $M$ is $L^{n}$-determined which together with (II) implies that

(III) if $m<\omega, a_{1}, \ldots, a_{m} \in M$ are different elements and $b_{1}, \ldots, b_{m} \in M$ are different elements, then $\left(M, a_{1}, \ldots, a_{m}\right) \equiv_{L}\left(M, b_{1}, \ldots, b_{m}\right)$ if and only if for all $i, j \in\{1, \ldots, m\}, M \models E\left(a_{i}, a_{j}\right) \Longleftrightarrow M \models E\left(b_{i}, b_{j}\right)$.

Hence $T h_{L}(M)$ has elimination of quantifiers and is $\omega$-categorical. Since $M$ is the limit of $\mathcal{A}$, conditions (ii) and (iii) in Lemma 2.9 are satisfied. This together with (I) implies that for every $m<\omega$, every choice of distinct $a_{1}, \ldots, a_{m} \in M$ and every $w \subseteq\{1, \ldots, m\}$, there is $b \in M$ such that $M \models E\left(a_{i}, b\right) \Longleftrightarrow i \in w$. Hence $M \models \varphi^{m}$ for every $1<m<\omega$. This implies that $M$ is the random graph $[12,20]$. It is well-known that $T h_{L}(M)$ is simple with SU-rank 1 and has trivial dependence [32]. In [26] it is shown that $T h_{L}(M)$ has the $k$-embedding of types property with respect to all generators, for every $1<k<\omega$. Note that since $M \models \varphi^{m}$ for every $1<m<\omega$, we can, for every $m<\omega$, find $a_{i}, b_{i} \in M$ for $i<m$ such that $M=E\left(a_{i}, b_{j}\right)$ if and only if $i \leq j$. Hence $M$ and $\mathcal{A}$ are not stable in $L^{n}$.

Corollary 5.4 implies that $T^{n}=\left\{\varphi^{n}\right\}$ has arbitrarily large finite models (all of which are isomorphic to substructures of $M$ ). This is nothing new, since the proof of the socalled 0-1 law for the random graph shows that, for every $1<n<\omega$, the number of graphs with universe $m=\{0, \ldots, m-1\}$ which satisfy $T^{n}$ divided by the number of all graphs with universe $m$ approaches 1 as $m \rightarrow \infty[12,20]$. At the core of the proof is a probabilistic argument ("What is the probability that $\varphi^{n}$ holds in a graph with universe $m$ ?"). The method in this example of showing that $\varphi^{n}$ has arbitrarily large finite models does not avoid the main idea, the probabilistic argument, in the proof of the 0-1 law. On the contrary, our approach has utilized this idea in a more general setting, but this is not evident in this paper since we don't discuss the proof of Theorem 5.1 or of Theorem 6.7, on which the former theorem relies.

Remark 5.6. It would be nice if we could specify some properties of complete $L^{n}$-theories (without speaking about limits of amalgamation classes) which, if they hold for such a theory, would allow us to derive the existence of a structure $M$ as in Theorem 5.1. However, while the notion of stability straightforwardly transfers from the context of complete $L$ theories to complete $L^{n}$-theories (in Section 3 ) the notions 'n-degenerate forking', 'SU-rank' and ' $n$-embedding of types property' involve the stability/simplicity theoretic notion of forking (or (in)dependence) and the author does not currently see a straightforward, or "natural", way of defining forking with respect to a complete $L^{n}$-theory (which, according to our definition, need not be complete in the usual sense). The notion of simplicity may, on the other hand, be straightforwardly transferred to the context of complete $L^{n}$-theories by saying that a complete $L^{n}$-theory is simple if no $L^{n}$-formula has the tree property (see [32]) in any model of the theory. However, the question remains whether simplicity, defined in this way, has any interesting consequences for $L^{n}$-theories.

\section{Structures on Which algebraic Closure forms a Pregeometry}

In this section we give a brief overview of the main results in [10], about the finite submodel property, which are stated as Theorems 6.6 and 6.7 below. Theorem 6.7 is used to prove the main result in [26] (stated as Theorem 5.1 in this survey). We will assume throughout this section that $M$ is an $\omega$-categorical $L$-structure such that the algebraic closure in $M$, 
denoted $\operatorname{acl}_{M}$, forms a pregeometry on $M$; see for instance [10] or [20] for a definition of a pregeometry. A consequence of $M$ being $\omega$-categorical is that for every finite $A \subseteq M$, $\operatorname{acl}_{M}(A)$ is finite. Moreover, in this context every subset $A \subseteq M$ has a dimension defined by

$$
\operatorname{dim}_{M}(A)=\inf \left\{|B|: B \subseteq A \text { and } A \subseteq \operatorname{acl}_{M}(B)\right\} .
$$

A type is called algebraic if it has only finitely many realizations.

Definition 6.1. Let $0<k<\omega$. We say that $M$ is polynomially $k$-saturated if there is a polynomial $P(x)$ such that for every $n_{0}<\omega$ there is a natural number $n \geq n_{0}$ and a finite substructure $N \subseteq M$ such that:

(1) $n \leq|N| \leq P(n)$.

(2) $N$ is algebraically closed (in $M$ ).

(3) Whenever $A \subseteq N, \operatorname{dim}_{M}(A)<k$ and $q(x) \in S_{1}^{L}(A, M)$ is non-algebraic, then there are distinct $b_{1}, \ldots, b_{n} \in N$ such that $M \models q\left(b_{i}\right)$ (i.e. $b_{i}$ realizes $q$ in $M$ ) for each $1 \leq i \leq n$.

Examples of structures on which the algebraic closure forms a pregeometry and which are polynomially $k$-saturated for every $0<k<\omega$ include the "infinite empty structure" (having only the relation '='), the random (bipartite) graph, infinite vector spaces, projective spaces and affine spaces over any finite field [10]. Another example is obtained by "independently" expanding a vector space (for instance) with the random graph [10].

We also have the following result from [10] which relates polynomial $k$-saturation to the finite submodel property.

Lemma 6.2. If $M$ is polynomially $k$-saturated for every $0<k<\omega$, then $M$ has the finite submodel property.

Assumption 6.3. From now on $\mathcal{L}$ is a first-order language such that $\mathcal{L}$ 's vocabulary is included in $L$ 's vocabulary, so $\mathcal{L} \subseteq L$. We suppose that $\operatorname{acl}_{M}$ coincides with acl $\operatorname{Mi\mathcal {L}}_{\text {(i.e. }}$ $\operatorname{acl}_{M}(A)=\operatorname{acl}_{M \uparrow \mathcal{L}}(A)$ for every $\left.A \subseteq M\right)$. Moreover, we assume that both $M$ and $M\lceil\mathcal{L}$ have elimination of quantifiers, where $M\lceil\mathcal{L}$ denotes the reduct of $M$ to $\mathcal{L}$. If these conditions are not fulfilled in the beginning, then we can just add new relation symbols to $L$ and $\mathcal{L}$ so that the resulting expansions satisfy these conditions and all previous assumptions about $M$.

Before going to the next definition we note that if $\operatorname{acl}_{M}$ and $\operatorname{acl}_{M \backslash \mathcal{L}}$ coincide and $\bar{a}, \bar{b} \in M$ satisfy exactly the same $\mathcal{L}$-formulas, then $\bar{a}$ is algebraically closed if and only if $\bar{b}$ is.

Definition 6.4. We say that $M$ satisfies the $k$-independence hypothesis over $\mathcal{L}$ if the following holds:

Whenever $A$ and $B$ are algebraically closed substructures of $M$ and

(1) $\operatorname{dim}_{M}(B) \leq k$,

(2) $\operatorname{dim}_{M}(A)<k$,

(3) $f: A \uparrow \mathcal{L} \rightarrow B \backslash \mathcal{L}$ is an $\mathcal{L}$-embedding (i.e. it preserves all atomic $\mathcal{L}$-formulas and negations of atomic $\mathcal{L}$-formulas), and

(4) if $A^{\prime} \subset A$ (proper inclusion) is an algebraically closed substructure then the restriction $f: A^{\prime} \rightarrow B$ is an $L$-embedding,

then there are an algebraically closed substructure $C \subseteq M$ and an $\mathcal{L}$-ismorphism $g: B \uparrow$ $\mathcal{L} \rightarrow C\lceil\mathcal{L}$ such that

(5) $g f: A \rightarrow C$ is an $L$-embedding, and

(6) for every algebraically closed substructure $B^{\prime} \subseteq B$ such that $f(A) \nsubseteq B^{\prime}, g: B^{\prime} \rightarrow C$ is an $L$-embedding. 
The definition of the $k$-independence hypothesis given above looks a bit different from the definition of it given in [10], but the two ways of defining the $k$-independence hypothesis are equivalent under Assumption 6.3. Below follow some examples which illustrate the $k$-independence hypothesis.

Examples 6.5. (a) The random graph: Let the vocabulary of $\mathcal{L}$ be $\{=\}$ and let the vocabulary of $L$ be $\{=, E\}$ where $E$ is a binary relation symbol. Let $M$ be the random graph in the language $L$ where $E$ is interpreted as the edge relation. Then $M$ and $M \uparrow \mathcal{L}$ have elimination of quantifiers and $\operatorname{acl}_{M}(A)=A$ and $\operatorname{dim}_{M}(A)=|A|$ for any $A \subseteq M$. Since any finite graph embedds into $M$ it follows that $M$ satisfies the $k$-independence hypothesis over $\mathcal{L}$ for every $k<\omega$. With the notation of the definition, the case when $\operatorname{dim}_{M}(A)=2$ is the most interesting. The reason is that $M$ has elimination of quantifiers in a language with only binary relations symbols and that dimension coincides with cardinality.

(b) The random structure: Let $\mathcal{L}$ be as in (a) and let the vocabulary of $L$ be $\left\{=, R_{1}, \ldots, R_{m}\right\}$ where $R_{i}$ are relation symbols of any arity. Let $M$ be the random structure in the language $L$, i.e. $M$ is the Fraïssé limit of the class of all finite $L$-structures. For the same reasons as in (a), $M$ satisfies the $k$-independence hypothesis over $\mathcal{L}$ for every $k<\omega$. However the verification becomes a little bit more interesting for $A$ of dimension $>2$ if $L$ contains relation symbols of arity greater than 2 .

(c) A vector space expanded with the bipartite random graph: Let $K$ be the class of all finite structures

$N=\left(V, P, E,+, f_{0}, f_{1}, 0\right)$ such that:

1. $V$, the universe of $N$, is a vector space over the field $F=\{0,1\}$.

2. $P$ is a unary relation.

3. $E$ is a binary relation symbol interpreted as an irreflexive and symmetric relation.

4. + is a binary function symbol interpreted as vector addition and the constant symbol 0 is interpreted as the zero vector.

5. $f_{i}(v)=i \cdot v$, for $i=0,1$ and any $v \in V$ (so $f_{i}$ represents scalar multiplication by $i$ ).

6. $N \models \forall x y(E(x, y) \rightarrow[[(P(x) \wedge \neg P(y)) \vee(\neg P(x) \wedge P(y))]])$.

7. $N=P(0)$.

It is easy to verify that $K$ is nonempty and has the hereditary property, the joint embedding property and the amalgamation property and is uniformly locally finite (see [20]). Hence the Fraïssé limit of $K$, which we call $M$, exists and is $\omega$-categorical with elimination of quantifiers. Since the reduct of $M$ to the language with vocabulary $\{=, P, E\}$ is the random bipartite graph, $M$ is not smoothly approximable [3].

Let $\mathcal{L} \subseteq L$ be the sublanguage which contains all symbols of $L$ except $P$ and $E$. Then $M\lceil\mathcal{L}$ is a vector space over a finite field, so $M\lceil\mathcal{L}$ has elimination of quantifiers. It is not hard to see, using quantifier elimination of $M$ and the fact that any structure in $K$ can be embedded into $M$ (since $M$ is the Fraissé limit of $K$ ), that $\operatorname{acl}_{M}(A)$ is linear span of $A$. Hence $\operatorname{acl}_{M}$ and $\operatorname{acl}_{M \backslash \mathcal{L}}$ coincide. Again using the fact that $M$ is the Fraisse limit of $K$ it follows that $M$ satisfies the $k$-independence hypothesis over $\mathcal{L}$, for every $k<\omega$.

(d) The random pyramid-free (3)-hypergraph: As shown in [10], the random pyramid-free (3)-hypergraph does not satisfy the 4-independence hypothesis over the language $\mathcal{L}$ with vocabulary $\{=\}$ (as opposed to the case of the random graph).

Having Assumption 6.3 in mind, we now state the two main results of [10].

Theorem 6.6. Suppose that $M \uparrow \mathcal{L}$ is polynomially $k$-saturated and that $M$ satisfies the $k$-independence hypothesis over $\mathcal{L}$. If $\varphi \in L$ is an unnested sentence, in which at most $k$ distinct variables occur, and $M \models \varphi$, then $\varphi$ has arbitrarily large finite models. 
Note that Theorem 6.6 only speaks about arbitrarily large finite models, but does not claim that these can be taken as substructures of $M$.

Theorem 6.7. Suppose that, for every $0<k<\omega, M\lceil\mathcal{L}$ is polynomially $k$-saturated and that $M$ satisfies the $k$-independence hypothesis over $\mathcal{L}$. Then $M$ is polynomially $k$-saturated, for every $0<k<\omega$, and hence $M$ has the finite submodel property.

We say that $M$ has trivial (also called degenerate) algebraic closure if for every $A \subseteq M$, $\operatorname{acl}_{M}(A)=\bigcup_{a \in A} \operatorname{acl}_{M}(a)$. Examples of ( $\omega$-categorical) $M$ which are simple with SU-rank 1 and trivial algebraic closure include the random (bipartite) graph, the random structure and the random pyramid-free (3)-hypergraph. The following is a consequence of the first theorem:

Corollary 6.8. Suppose that $M$ is simple with SU-rank 1 and has trivial algebraic closure. If $\varphi \in L^{3}$ is unnested and $M \models \varphi$ then $\varphi$ has arbitrarily large finite models.

The assumption that $M$ satisfies the $k$-independence hypothesis (over $\mathcal{L}$ ) in the previous two theorems is used in the probabilistic argument at the core of the proofs. It generalizes the argument used when showing that the random graph (or random structure) satisfies a $0-1$ law with the uniform probability measure. The proofs of Theorems 6.6 and 6.7 do not however lead to 0-1 laws in general, with the uniform probability measure.

It seems that without assuming any kind of independence we get into a difficult situation with respect to proving or disproving the finite submodel property, as witnessed by the complete theory of the random pyramid-free (3)-hypergraph (example (d) above). It is $\omega$ categorical with elimination of quantifiers, simple with SU-rank 1, has trivial dependence and trivial algebraic closure. However, for all $k \geq 4$, it does not satisfy the $k$-independence hypothesis over (the only proper sublanguage) $\mathcal{L}$ with vocabulary $\{=\}[10]$. Neither does it have the $n$-embedding of types property for any $n \geq 4$, nor does it have have the $n$ amalgamation property for any $n \geq 4[25,26]$. It is an open problem whether the random pyramid-free (3)-hypergraph has the finite submodel property or not.

\section{Questions And Problems}

In connection with the approach exposed in this paper one may of course ask many questions, some of which are stated below.

(1) Can we find "natural" amalgamation properties and stability/simplicity theoretic properties for $L^{n}$-theories $T$ (or other fragments of first-order logic) which imply the existence of an infinite model $M$ of $T$ with the finite model property (i.e. every sentence which is true in $M$ is true in a finite model), for other classes of theories $T$ than those that fit into the framework presented here (in sections 1-3)?

(2) In particular, can we find "natural" amalgamation properties and stability/simplicity theoretic properties for "simple" $L^{n}$-theories $T$ (without a stable amalgamation class) which guarantee that $T$ has a model such as $M$ in Theorem 5.1?

(3) Can stronger upper bounds than recursive (exponentional, polynomial etc.) on the size of the least model be obtained for some interesting classes of theories?

(4) Are there other approaches, than the one presented here, towards understanding when (arbitrarily large) finite models exist and when a recursive (or better) upper bound of the smallest model exist, in terms of the number of $L^{n}$-types, for instance?

(5) Can one derive the conclusions of Theorem 5.1 from a weaker assumption than that forking is trivial?

(6) Can the approach in Section 9, about structures on which algebraic closure forms a pregeometry, be helpful for understanding $L^{n}$-theories (or, say, theories in a language with a finite bound on the quantifier rank)?

(7) The random graph fits within the framework presented in sections 5 and 6 as well as within the framework of 'asymptotic classes' and 'measurable structures' [28, 13, 14, 15]. 
Do the two approaches have anything in common? If 'yes', can both approaches together enrich our knowledge about relationships between infinite structures and classes of finite structures.

(8) Does the random pyramid-free (3)-hypergraph (Example 6.5 (d)) have the finite submodel property?

\section{REFERENCES}

[1] J. Baldwin, Finite and infinite model theory - a historical perspective, Logic Journal of the IGPL, Vol. 8 (2000) 605-628.

[2] J. Baldwin, O. Lessmann, Amalgamation properties and finite models in $L^{n}$-theories, Archive for Mathematical Logic, Vol. 41 (2002) 155-167.

[3] G. Cherlin, E. Hrushovski, Finite structures with few types, Annals of Mathematics Studies 152, Princeton University Press 2003.

[4] G. Cherlin, L. Harrington, A. H. Lachlan, $\aleph_{0}$-categorical, $\aleph_{0}$-stable structures, Annals of Pure and Applied Logic, 28 (1985) 103-135.

[5] A. Dawar, Types and indiscernibles in finite models, in Logic Colloquium '95, Lecture Notes in Logic, Vol. 11, Springer.

[6] A. Dawar, S. Lindell, S. Weinstein, Infinitary logic and inductive definability over finite structures, Information and Computation, Vol. 119 (1995) 160-175.

[7] T. De Piro, B. Kim, The geometry of 1-based minal types, Transactions of the Americal Mathematical Society, Vol. 355 (2003) 4241-4263.

[8] M. Djordjević, Stability theory in finite variable logic, Ph.D. thesis, Uppsala University 2000.

[9] M. Djordjević, Finite variable logic, stability and finite models, The Journal of Symbolic Logic, Vol. 66 (2001) 837-858.

[10] M. Djordjević, The finite submodel property and $\omega$-categorical expansions of pregeometries, Annals of Pure and Applied Logic Vol. 139 (2006) 201-229.

[11] M. Djordjević, Finite satisfiability and $\aleph_{0}$-categorical structures with trivial dependence, The Journal of Symbolic Logic, Vol. 71 (2006) 810-830.

[12] H-D. Ebbinghaus, J. Flum, Finite Model Theory, Second Edition, Springer Verlag, 1999.

[13] R. Elwes, Dimension and measure in finite first order structures, PhD thesis, University of Leeds, 2005.

[14] R. Elwes, Asymptotic classes of finite structures, The Journal of Symbolic Logic, Vol. 72 (2007) 418438.

[15] R. Elwes, H.D. Macpherson, A survey of asymptotic classes and measurable structures, in Model theory and applications to algebra and analysis. Eds. Z. Chatzidakis, H.D. Macpherson, A. Pillay, A.J. Wilkie, Cambridge University Press, to appear.

[16] M. Grohe, Finite variable logic in descriptive complexity theory, The Bulletin of Symbolic Logic, Vol. 4 (1998) 345-398.

[17] M. Grohe, Large finite structures with few $L^{k}$-types, Information and Computation, Vol. 179 (2002) $250-278$.

[18] B. Hart, B. Kim, A. Pillay, Coordinatisation and canonical bases in simple theories, The Journal of Symbolic Logic, Vol. 65 (2000) 293-309.

[19] S. Hedman, Finitary axiomatizations of strongly minimal theories, Ph.D. thesis, University of Illinois at Chicago, 1999.

[20] W. Hodges, Model theory, Cambridge University Press 1993.

[21] T. Hyttinen, On stability in finite models, Archive for Mathematical Logic, Vol. 39 (2000) 89-102.

[22] T. Hyttinen, Canonical finite diagrams and quantifier elimination, Mathmatical Logic Quarterly, Vol. 48 (2002) 533-554.

[23] N. Immerman, Upper and lower for first order expressibility, Journal och Computer and Systems Sciences, Vol. 25 (1982) 76-98.

[24] W. M. Kantor, M. W. Liebeck, H. D. Macpherson, $\aleph_{0}$-categorical structures smoothly approximated by finite substructures, Proceedings of the London Mathematical Society, Vol. 59 (1989) 439-463.

[25] B. Kim, A.S. Kolesnikov, A. Tsuboi, Generalized amalgamation and $n$-simplicity, Annals of Pure and Applied Logic, Vol. 155 (2008) 97-114.

[26] V. Koponen, Independence and the finite submodel property, Annals of Pure and Applied Logic, Vol. 158 (2009) 58-79.

[27] A. H. Lachlan, Stable finitely homogeneous structures: a survey, in B. T. Hart, A. H. Lachlan, M. A. Valeriote, editors, Algebraic model theory, Kluwer Academic Publishers (1997) 145-159.

[28] H.D. Macpherson, C. Steinhorn, One-dimensional asymptotic classes of finite structures, Transactions of the American Mathematical Society, Vol. 360 (2008) 411-448. 
[29] B. Poizat, Deux ou trois choses que je sais de $L_{n}$, The Journal of Symbolic Logic, Vol. 47 (1982) 641-658.

[30] E. Rosen, Some aspects of model theory and finite structures, The Bulletin of Symbolic Logic, Vol. 8 (2002) 380-403.

[31] S. Shelah, Classification Theory, North-Holland 1990.

[32] F. O. Wagner, Simple theories, Kluwer Academic Publishers 2000.

[33] B. Zilber, Uncountably categorical structures, AMS translations of mathematical monographs 117 (1993).

Department of Mathematics, Uppsala University, Box 480, 75106 Uppsala, Sweden

E-mail address: vera@math.uu.se 